Document downloaded from:

http://hdl.handle.net/10251/121735

This paper must be cited as:

Behl, R.; Cordero Barbero, A.; Motsa, SS.; Torregrosa Sánchez, JR. (2018). An eighth-order family of optimal multiple root finders and its dynamics. Numerical Algorithms. 77(4):12491272. https://doi.org/10.1007/s11075-017-0361-6

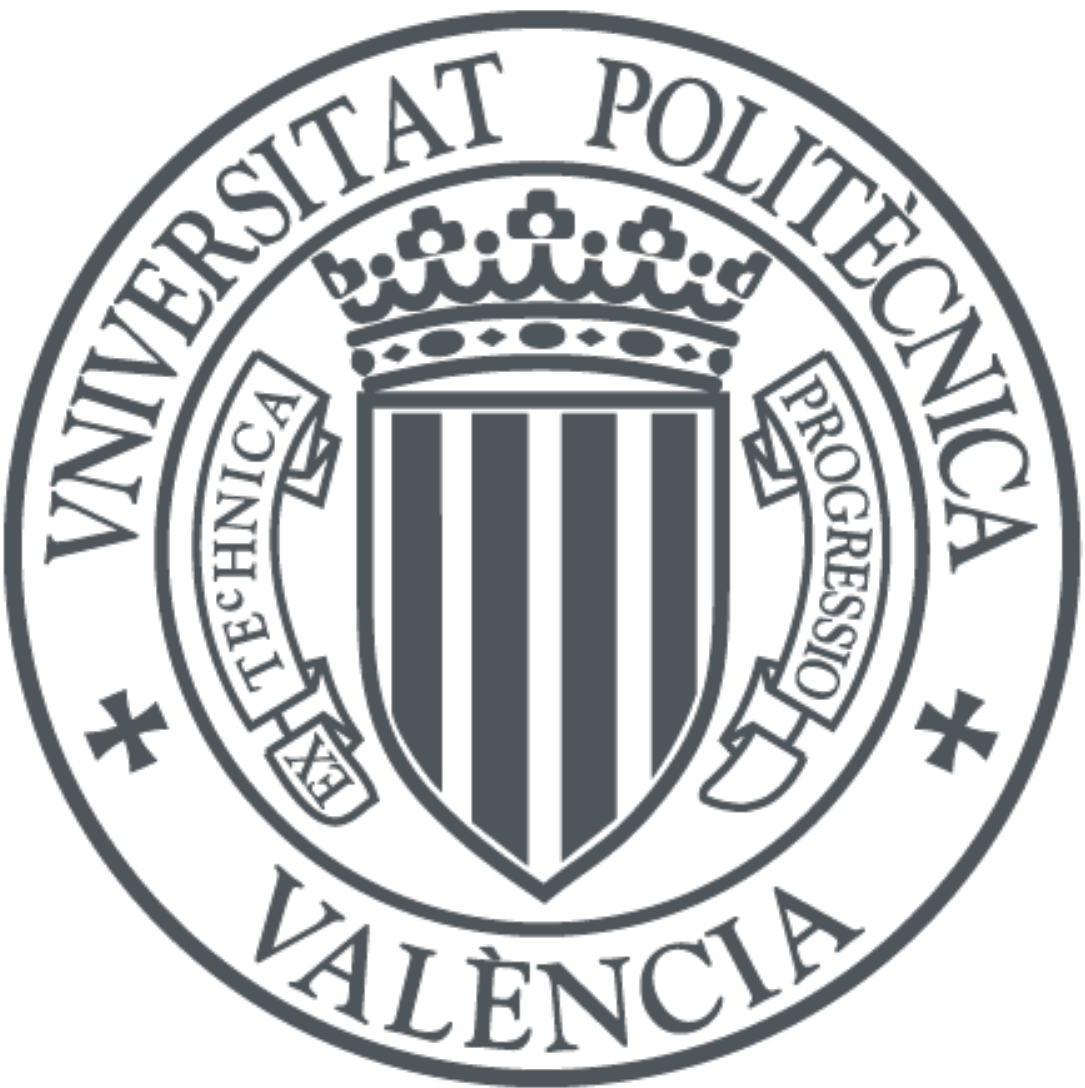

The final publication is available at

http://doi.org/10.1007/s11075-017-0361-6

Copyright Springer-Verlag

Additional Information 


\title{
An eighth-order family of optimal multiple-root finders and its dynamics
}

\author{
Ramandeep Behl · Alicia Cordero · Sandile S. Motsa · Juan R. \\ Torregrosa
}

Received: date / Accepted: date

\begin{abstract}
There is a very small number of higher-order iteration functions for multiple zeros whose order of convergence is greater than four. Some scholars have tried to propose optimal eighth-order methods for multiple zeros. But, unfortunately, they did not get success in this direction and attained only sixth-order convergence. So, as far as we know, there is not a single optimal eighth-order iteration function in the available literature that will work for multiple zeros. Motivated and inspired by this fact, we present an optimal eighth-order iteration function for multiple zeros. An extensive convergence study is discussed in order to demonstrate the optimal eighth-order convergence of the proposed scheme. In addition, we also demonstrate the applicability of our proposed scheme on real life problems and illustrate that the proposed methods are more efficient among the available multiple root finding techniques. Finally, dynamical study of the proposed schemes also confirms the theoretical results.
\end{abstract}

Keywords Nonlinear equations, optimal iterative methods, multiple roots, efficiency index, Kung-Traub conjecture.

\section{Introduction}

Construction of higher-order and optimal, in the sense of Kung-Traub conjecture [1], iteration functions, free from secondorder derivatives, is always required for the multiple zeros of univariate function of the form $f(x)=0$, which partake scientific, engineering, economic modeling, applied science and other various models. The well known second-order modified Newton's method (also known as Rall's method) is one of the simplest and most popular iteration functions for obtaining multiple zeros of univariate function, which is defined as follows:

$$
x_{n+1}=x_{n}-m \frac{f\left(x_{n}\right)}{f^{\prime}\left(x_{n}\right)},
$$

This research was partially supported by Ministerio de Economía y Competitividad MTM2014-52016-C2-2-P and Generalitat Valenciana PROMETEO/2016/089.

Ramandeep Behl

School of Mathematics, Statistics and Computer Sciences, University of KwaZulu-Natal,

Private Bag X01, Scottsville 3209, Pietermaritzburg, South Africa

Alicia Cordero

Instituto Universitario de Matemática Multidisciplinar,

Universitat Politècnica de València, Cno. de Vera s/n, 46022-València, Spain

Sandile S. Motsa

Mathematics Department, University of Swaziland, Private Bag 4, Kwaluseni, M201, Swaziland

School of Mathematics, Statistics and Computer Sciences, University of KwaZulu-Natal,

Private Bag X01, Scottsville 3209, Pietermaritzburg, South Africa

Juan R. Torregrosa

Instituto Universitario de Matemática Multidisciplinar,

Universitat Politècnica de València, Cno. de Vera s/n, 46022-València, Spain 
where $m$ is the multiplicity of the required zero and known in advance. However, this is a one-point iteration function. There are several problems related to one-point iteration functions regarding the convergence, efficiency and involvement of higherorder derivative which can be seen in some well-known standard text books Ostrowski [2], Traub [3] and Petković et al. [4].

Therefore, scholars from the worldwide turn towards multipoint iteration functions which not only overcome the theoretical limitation of one-point iteration functions but also belong to one of the most important class of iterative methods. However, we have a very small number of optimal fourth-order iteration functions $[5,6,8,9,10,11,12,13]$ for multiple zeros to date and other non-optimal ones as $[9,14]$.

Recently, Geum et al. in [15], presented a non-optimal family of two-point sixth-order methods to find multiple zeros, given as follows:

$$
\begin{aligned}
y_{n} & =x_{n}-m \frac{f\left(x_{n}\right)}{f^{\prime}\left(x_{n}\right)}, m>1, \\
x_{n+1} & =y_{n}-Q\left(u_{n}, s_{n}\right) \frac{f\left(y_{n}\right)}{f^{\prime}\left(y_{n}\right)},
\end{aligned}
$$

where $u_{n}=\sqrt[m]{\frac{f\left(y_{n}\right)}{f\left(x_{n}\right)}}, s_{n}=\sqrt[m-1]{\frac{f^{\prime}\left(y_{n}\right)}{f^{\prime}\left(x_{n}\right)}}$ and $Q$ is an analytic function in a neighborhood of $(0,0)$. The problem with this scheme is that it does not work for simple zeros $(m=1)$.

Very recently, another non-optimal family of three-point sixth-order methods for multiple zeros was proposed by Geum et al. [16], and it is given by

$$
\begin{aligned}
y_{n} & =x_{n}-m \frac{f\left(x_{n}\right)}{f^{\prime}\left(x_{n}\right)}, m \geq 1, \\
w_{n} & =y_{n}-m G\left(u_{n}\right) \frac{f\left(x_{n}\right)}{f^{\prime}\left(x_{n}\right)}, \\
x_{n+1} & =w_{n}-m K\left(u_{n}, v_{n}\right) \frac{f\left(x_{n}\right)}{f^{\prime}\left(x_{n}\right)},
\end{aligned}
$$

where $u_{n}=\sqrt[m]{\frac{f\left(y_{n}\right)}{f\left(x_{n}\right)}}$ and $v_{n}=\sqrt[m]{\frac{f\left(w_{n}\right)}{f\left(x_{n}\right)}}$. The weight functions $G: \mathbb{C} \rightarrow \mathbb{C}$ and $K: \mathbb{C}^{2} \rightarrow \mathbb{C}$ are analytic in a neighborhood of 0 and $(0,0)$, respectively.

It is clear from the above discussion that several scholars from the worldwide have tried to obtain an optimal eighth-order iteration function for multiple zeros. But, no success was achieved in this direction and the highest attained order was six, which was mentioned above. So, this means there is not a single research article where any scholar claims optimal eighthorder convergent method to this date, according to our knowledge. As we know, the optimal iterative methods have more importance than the non-optimal ones, regarding their efficiency and faster convergence.

Keeping all these points in our mind, we try to solve this problem which has been pending for several years. So, we present an optimal eighth-order scheme for multiple zeros. The derivation of the proposed scheme is based on weight function approach and its extension to multiple zeros. In the case of simple zeros, the scheme proposed by Artidiello et al. [17] can be obtained as special case of our proposed scheme by assigning $(m=1)$. In addition, our proposed methods not only give the faster convergence but also have smaller residual error. Our proposed scheme only consumes four function evaluations $\left(f\left(x_{n}\right), f^{\prime}\left(x_{n}\right) f\left(y_{n}\right)\right.$ and $\left.f\left(z_{n}\right)\right)$ per full iteration which satisfies the classical Kung-Traub conjecture. We have demonstrated the usefulness of the proposed methods by performing several applied science problems for numerical tests and observed in each example that our methods have far better numerical results, than the existing sixth-order methods (in terms of smaller error difference between two consecutive iterations and smaller residual errors). Further, the dynamical study of these methods also supports the theoretical aspects.

The rest of the paper is organized as follows: Section 2 is devoted to the design of the new class of iterative methods, the analysis of its convergence (proving the optimal eighth-order of convergence) and the definition of several special cases that will be tested in the following sections. In Section 3, some of these particular cases have been used to estimate the solution of four real-life problems, by using both numerical and dynamical tools. Finally, some conclusions are stated. 


\section{Development of the family}

In this section, we propose a new eighth-order scheme

$$
\begin{aligned}
y_{n} & =x_{n}-m \frac{f\left(x_{n}\right)}{f^{\prime}\left(x_{n}\right)}, \\
z_{n} & =y_{n}-\frac{f\left(x_{n}\right)}{f^{\prime}\left(x_{n}\right)} u_{n} Q\left(h_{n}\right), \\
x_{n+1} & =z_{n}-\frac{f\left(x_{n}\right)}{f^{\prime}\left(x_{n}\right)} u_{n} t_{n} G\left(h_{n}, t_{n}\right),
\end{aligned}
$$

where the weight functions $Q: \mathbb{C} \rightarrow \mathbb{C}$ and $G: \mathbb{C}^{2} \rightarrow \mathbb{C}$ are analytic functions in a neighborhoods of $(0)$ and $(0,0)$, respectively, with $u_{n}=\left(\frac{f\left(y_{n}\right)}{f\left(x_{n}\right)}\right)^{\frac{1}{m}}, h_{n}=\frac{u_{n}}{a_{1}+a_{2} u_{n}}$ and $t_{n}=\left(\frac{f\left(z_{n}\right)}{f\left(y_{n}\right)}\right)^{\frac{1}{m}}$, being $a_{1}$ and $a_{2}$ are complex non zero parameters.

In the next Theorem 1, we demonstrate that the order of convergence of the proposed scheme will reach at eight without using any additional function evaluations. It is interesting to note that the two weight functions $Q$ and $G$ play a significant role in the construction of desired optimal eighth-order convergence.

Theorem 1 Let $x=\xi$ be a multiple zero with multiplicity $m$ of an analytic function $f: \mathbb{C} \rightarrow \mathbb{C}$ in the region enclosing the multiple zero $\xi$. Then, the iterative schemes defined by (4) have eighth-order convergence when the following conditions hold

$$
\left\{\begin{array}{l}
Q(0)=m, \quad Q^{\prime}(0)=2 a_{1} m, \quad G_{00}=m, \quad G_{10}=2 a_{1} m, \quad G_{01}=m, \\
G_{20}=Q^{\prime \prime}(0)+2 a_{1}^{2} m, \quad G_{11}=4 a_{1} m, \quad G_{30}=Q^{(3)}(0)+6 a_{1} Q^{\prime \prime}(0)-24 m a_{1}^{3}-12 m a_{1}^{2} a_{2},
\end{array}\right.
$$

where $G_{i j}=\frac{\partial^{i+j} G}{\partial h^{i} \partial t^{j}}(0,0), i, j \in\{0,1,2,3,4\}$.

Proof Let us consider $x=\xi$ be a multiple zero of $f(x)$. In addition, expanding $f\left(x_{n}\right)$ and $f^{\prime}\left(x_{n}\right)$ about $x=\xi$ by the Taylor's series (with the help of computer algebra software Mathematica), we obtain

$$
f\left(x_{n}\right)=\frac{f^{(m)}(\xi)}{m !} e_{n}^{m}\left(1+c_{1} e_{n}+c_{2} e_{n}^{2}+c_{3} e_{n}^{3}+c_{4} e_{n}^{4}+c_{5} e_{n}^{5}+c_{6} e_{n}^{6}+c_{7} e_{n}^{7}+c_{8} e_{n}^{8}+O\left(e_{n}^{9}\right)\right)
$$

and

$$
\begin{aligned}
f^{\prime}\left(x_{n}\right)= & \frac{f^{m}(\xi)}{(m) !} e_{n}^{m-1}\left(m+c_{1}(m+1) e_{n}+c_{2}(m+2) e_{n}^{2}+c_{3}(m+3) e_{n}^{3}+c_{4}(m+4) e_{n}^{4}+c_{5}(m+5) e_{n}^{5}\right. \\
& \left.+c_{6}(m+6) e_{n}^{6}+c_{7}(m+7) e_{n}^{7}+c_{8}(m+8) e_{n}^{8}+O\left(e_{n}^{9}\right)\right)
\end{aligned}
$$

where $e_{n}=x_{n}-\xi$.

By using the above equations (6) and (7), we obtain

$$
y_{n}-\xi=\frac{c_{1} e_{n}^{2}}{m}+\sum_{i=1}^{6} \Omega_{i} e_{n}^{i+2}+O\left(e_{n}^{9}\right),
$$

where $\Omega_{i}=\Omega_{i}\left(m, c_{1}, c_{2}, \ldots, c_{8}\right)$ are given in terms of $m, c_{2}, c_{3}, \ldots, c_{8}$ with explicitly written three coefficients $\Omega_{1}=$ $\frac{2 c_{2} m-c_{1}^{2}(m+1)}{m^{2}}, \Omega_{2}=\frac{1}{m^{3}}\left\{3 c_{3} m^{2}+c_{1}^{3}(m+1)^{2}-c_{2} c_{1} m(3 m+4)\right\}$ and $\Omega_{3}=\frac{1}{m^{4}}\left\{c_{1}^{4}(m+1)^{3}-2 c_{2} c_{1}^{2} m\left(2 m^{2}+5 m+3\right)+\right.$ $\left.2 c_{3} c_{1} m^{2}(2 m+3)+2 m^{2}\left(c_{2}^{2}(m+2)-2 c_{4} m\right)\right\}$, etc.

With the help of Taylor Series expansion, we further obtain

$$
\begin{aligned}
f\left(y_{n}\right)= & f^{(m)}(\xi) e_{n}^{2 m}\left[\frac{\left(\frac{c_{1}}{m}\right)^{m}}{m !}+\frac{\left(2 c_{2} m-c_{1}^{2}(m+1)\right)\left(\frac{c_{1}}{m}\right)^{m} e_{n}}{c_{1} m !}+\left(\frac{c_{1}}{m}\right)^{1+m} \frac{1}{2 m ! c_{1}^{3}}\left\{\left(3+3 m+3 m^{2}+m^{3}\right) c_{1}^{4}\right.\right. \\
& \left.\left.-2 m\left(2+3 m+2 m^{2}\right) c_{1}^{2} c_{2}+4(-1+m) m^{2} c_{2}^{2}+6 m^{2} c_{1} c_{3}\right\} e_{n}^{2}+\sum_{i=0}^{5} \bar{\Omega}_{i} e_{n}^{i+3}+O\left(e_{n}^{9}\right)\right]
\end{aligned}
$$

and

$$
u_{n}=\frac{c_{1} e_{n}}{m}+\frac{\left(2 c_{2} m-c_{1}^{2}(m+2)\right) e_{n}^{2}}{m^{2}}+\eta_{1} e_{n}^{3}+\eta_{2} e_{n}^{4}+\eta_{3} e_{n}^{5}+O\left(e_{n}^{6}\right),
$$


where $\eta_{1}=\frac{1}{2 m^{3}}\left[c_{1}^{3}\left(2 m^{2}+7 m+7\right)+6 c_{3} m^{2}-2 c_{2} c_{1} m(3 m+7)\right], \eta_{2}=-\frac{1}{6 m^{4}}\left[c_{1}^{4}\left(6 m^{3}+29 m^{2}+51 m+34\right)-6 c_{2} c_{1}^{2} m\left(4 m^{2}+\right.\right.$ $\left.16 m+17)+12 c_{3} c_{1} m^{2}(2 m+5)+12 m^{2}\left(c_{2}^{2}(m+3)-2 c_{4} m\right)\right]$ and $\eta_{3}=\frac{1}{24 m^{5}}\left[-24 m^{3}\left(c_{2} c_{3}(5 m+17)-5 c_{5} m\right)+\right.$ $12 c_{3} c_{1}^{2} m^{2}\left(10 m^{2}+43 m+49\right)+12 c_{1} m^{2}\left\{c_{2}^{2}\left(10 m^{2}+47 m+53\right)-2 c_{4} m(5 m+13)\right\}-4 c_{2} c_{1}^{3} m\left(30 m^{3}+163 m^{2}+306 m+\right.$ $\left.209)+c_{1}^{5}\left(24 m^{4}+146 m^{3}+355 m^{2}+418 m+209\right)\right]$.

With the help of above expression (10), we have

$$
h_{n}=\frac{c_{1}}{m a_{1}} e_{n}+\sum_{i=1}^{4} \Gamma_{i} e_{n}^{i+1}+O\left(e_{n}^{6}\right)
$$

where $\Gamma_{i}=\Gamma_{i}\left(a_{1}, a_{2}, m, c_{1}, c_{2}, \ldots, c_{8}\right)$ are given in terms of $a_{1}, a_{2}, m, c_{1}, c_{2}, \ldots, c_{8}$ with explicitly written two coefficients $\Gamma_{1}=-\frac{a_{2} c_{1}^{2}+a_{1}\left((2+m) c_{1}^{2}-2 m c_{2}\right)}{m^{2} a_{1}^{2}}, \Gamma_{2}=\frac{1}{2 m^{3} a_{1}^{3}}\left[2 a_{2}^{2} c_{1}^{3}+4 a_{1} a_{2} c_{1}\left((2+m) c_{1}^{2}-2 m c_{2}\right)+a_{1}^{2}\left\{\left(7+7 m+2 m^{2}\right) c_{1}^{3}-2 m(7+\right.\right.$ $\left.\left.3 m) c_{1} c_{2}+6 m^{2} c_{3}\right\}\right]$.

It is clear from (11) that $h_{n}$ is of order $e_{n}$. Therefore, we can expand weight function $Q\left(h_{n}\right)$ in the neighborhood of origin by Taylor series expansion up to fifth-order term for the eighth order convergence as follow:

$$
Q\left(h_{n}\right)=Q(0)+Q^{\prime}(0) h_{n}+\frac{1}{2 !} Q^{\prime \prime}(0) h_{n}^{2}+\frac{1}{3 !} Q^{(3)}(0) h_{n}^{3}+\frac{1}{4 !} Q^{(4)}(0) h_{n}^{4}+\frac{1}{5 !} Q^{(5)}(0) h_{n}^{5} .
$$

By inserting the expressions (6) - (12) in the second substep of the proposed scheme (4), we have

$$
\begin{aligned}
z_{n}-\xi= & \frac{(m-Q(0)) c_{1}}{m^{2}} e_{n}^{2}+\frac{2 m(m-Q(0)) a_{1} c_{2}-\left(Q^{\prime}(0)+\left(m+m^{2}-3 Q(0)-m Q(0)\right) a_{1}\right) c_{1}^{2}}{m^{3} a_{1}} e_{n}^{3} \\
& +\sum_{l=1}^{5} \Delta_{l} e_{n}^{l+3}+O\left(e_{n}^{9}\right) .
\end{aligned}
$$

where $\Delta_{l}=\Delta_{l}\left(Q(0), Q^{\prime}(0), Q^{\prime \prime}(0), Q^{(3)}(0), Q^{(4)}(0), m, a_{1}, a_{2}, c_{1}, c_{2}, \ldots, c_{8}\right), l=1,2,3,4,5$.

In order to obtain fourth-order convergence, the coefficient of $e_{n}^{2}$ and $e_{n}^{3}$ simultaneously are equaled to zero. That is possible only for the following values of $Q(0)$ and $Q^{\prime}(0)$, which can be calculated from the expression (13):

$$
Q(0)=m, \quad Q^{\prime}(0)=2 m a_{1} .
$$

By using the above expression (14), we have

$$
z_{n}-\xi=\frac{\left(m(9+m) a_{1}^{2}-Q^{\prime \prime}(0)+4 m a_{1} a_{2}\right) c_{1}^{3}-2 m^{2} a_{1}^{2} c_{1} c_{2}}{2 m^{4} a_{1}^{2}} e_{n}^{4}+\sum_{s=1}^{4} P_{s} e_{n}^{s+4}+O\left(e_{n}^{9}\right) .
$$

where $P_{s}=P_{s}\left(Q^{\prime \prime}(0), Q^{(3)}(0), Q^{(4)}(0), m, a_{1}, a_{2}, c_{1}, c_{2}, \ldots, c_{8}\right), s=1,2,3,4$.

Now, again by using the Taylor series expansion, we have

$$
f\left(z_{n}\right)=f^{(m)}(\xi) e_{n}^{4 m}\left[\frac{2^{-m}}{m !}\left(\frac{\left(m(9+m) a_{1}^{2}-Q^{\prime \prime}(0)+4 m a_{1} a_{2}\right) c_{1}^{3}-2 m^{2} a_{1}^{2} c_{1} c_{2}}{m^{4} a_{1}^{2}}\right) m+\sum_{s=1}^{5} \bar{P}_{s} e_{n}^{s}+O\left(e_{n}^{6}\right)\right]
$$

and

$$
t_{n}=\frac{\left(m(9+m) a_{1}^{2}-Q^{\prime \prime}(0)+4 m a_{1} a_{2}\right) c_{1}^{2}-2 m^{2} a_{1}^{2} c_{2}}{2 m^{3} a_{1}^{2}} e_{n}^{2}+\lambda_{1} e_{n}^{3}+\lambda_{2} e_{n}^{4}+\lambda_{3} e_{n}^{5}+O\left(e_{n}^{6}\right),
$$

where $\lambda_{1}=\frac{1}{3 m^{4} a_{1}^{3}}\left[3 Q^{\prime \prime}(0) a_{2} c_{1}^{3}-12 m a_{1}^{2} a_{2} c_{1}\left((3+m) c_{1}^{2}-2 m c_{2}\right)+3 a_{1}\left(\left((3+m) Q^{\prime \prime}(0)-2 m a_{2}^{2}\right) c_{1}^{3}-2 m Q^{\prime \prime}(0) c_{1} c_{2}\right)-\right.$ $\left.m a_{1}^{3}\left\{\left(49+27 m+2 m^{2}\right) c_{1}^{3}-6 m(9+m) c_{1} c_{2}+6 m^{2} c_{3}\right\}\right]$.

It is clear from (11) and (17) that $h_{n}$ and $t_{n}$ are of order $e_{n}$ and $e_{n}^{2}$, respectively. Therefore, we can expand weight function $G(h, t)$ in the neighborhood of origin $(0,0)$ by Taylor series expansion up to fourth-order terms as follow:

$$
\begin{aligned}
G\left(h_{n}, t_{n}\right) & =G_{00}+G_{10} h_{n}+G_{01} t_{n}+\frac{1}{2 !}\left(G_{20} h_{n}^{2}+2 G_{11} h_{n} t_{n}+G_{02} t_{n}^{2}\right)+\frac{1}{3 !}\left(G_{30} h_{n}^{3}+3 G_{21} h_{n}^{2} t_{n}+3 G_{12} h_{n} t_{n}^{2}+G_{03} t_{n}^{3}\right) \\
& +\frac{1}{4 !}\left(G_{40} h_{n}^{4}+4 G_{31} h_{n}^{3} t_{n}+6 G_{22} h_{n}^{2} t_{n}^{2}+4 G_{13} h_{n} t_{n}^{3}+G_{04} t_{n}^{4}\right)
\end{aligned}
$$

where $G_{i j}=\left.\frac{\partial^{i+j}}{\partial h^{i} \partial t^{j}} G(h, t)\right|_{(0,0)}, i, j \in\{0,1,2,3,4\}$. 
By using the expression (6) - (18), we have

$$
e_{n+1}=\frac{c_{1}\left(G_{00}-m\right)\left(2 a_{1}^{2} c_{2} m^{2}+c_{1}^{2}\left(a_{1}^{2}(-m)(m+9)-4 a_{2} a_{1} m+Q^{\prime \prime}(0)\right)\right)}{2 a_{1}^{2} m^{5}} e_{n}^{4}+\sum_{i=1}^{4} K_{i} e_{n}^{i+4}+O\left(e_{n}^{9}\right)
$$

where $K_{i}=K_{i}\left(m, a_{1}, a_{2}, Q(0), Q^{\prime}(0), Q^{\prime \prime}(0), Q^{(3)}(0), c_{1}, c_{2}, \ldots, c_{8}\right), i=1,2,3,4$.

It is clear from the above error expression (19) that we will obtain at least fifth-order convergence if we choose $G_{00}=m$. In addition, we will use this value $G_{00}=m$ in $K_{1}=0$. Then, we will obtain

$$
G_{10}=2 a_{1} m
$$

By using $G_{00}=m$ and the above expression (20) in $K_{2}=0$, we have

$$
G_{01}-m=0, \quad a_{1}^{2} m\left\{m(m+11)-G_{01}(m+9)\right\}+4 a_{2} a_{1} m\left(m-G_{01}\right)+G_{01} Q^{\prime \prime}(0)-G_{20} m=0,
$$

which further yield

$$
G_{01}=m, \quad G_{20}=2 a_{1}^{2} m+Q^{\prime \prime}(0) .
$$

By inserting $G_{00}=m$ and expressions (20) and (22) in $K_{2}=0$, we obtain the following two independent expressions

$$
\left(G_{11}-4 a_{1} m\right)=0, \quad-G_{30} m+3 G_{11} Q^{\prime \prime}(0)+m Q^{(3)}(0)+12 m^{2}(7+m) a_{1}^{3}-6 m a_{1}\left(Q^{\prime \prime}(0)+2 G_{11} a_{2}\right)-3 m a_{1}^{2}\left(G_{11}(9+m)-12 m a_{2}\right)=0,
$$

which leads us

$$
G_{11}=4 a_{1} m \quad G_{30}=Q^{(3)}(0)+6 a_{1} Q^{\prime \prime}(0)-24 m a_{1}^{3}-12 m a_{1}^{2} a_{2} .
$$

By using the value of $G_{00}=m$, the expressions (20), (22) and (24) in the expression (15), we will obtain the final and optimal asymptotic error constant

$$
\begin{aligned}
e_{n+1} & =\frac{c_{1}\left[\left(Q^{\prime \prime}(0)-m(9+m) a_{1}^{2}-4 m a_{1} a_{2}\right) c_{1}^{2}+2 m^{2} a_{1}^{2} c_{2}\right]}{48 m^{11} a_{1}^{6}}\left[\left\{G_{40} m^{2}-6 G_{21} m Q^{\prime \prime}(0)+3 G_{02} Q^{\prime \prime}(0)^{2}-m^{2} Q^{(4)}(0)\right.\right. \\
& +m^{2}\left(3 G_{02}(9+m)^{2}-2 m\left(431+102 m+7 m^{2}\right)\right) a_{1}^{4}+24 m^{2}\left(G_{02}(9+m)-m(23+3 m)\right) a_{1}^{3} a_{2}-8 m a_{1}\left(m Q^{(3)}(0)\right. \\
& \left.-3\left(G_{21} m+\left(-G_{02}+m\right) Q^{\prime \prime}(0)\right) a_{2}\right)+6 m a_{1}^{2}\left(G_{21} m(9+m)+\left(-G_{02}(9+m)+m(17+m)\right) Q^{\prime \prime}(0)+4\left(2 G_{02}\right.\right. \\
& \left.\left.-3 m) m a_{2}^{2}\right)\right\} c_{1}^{4}-12 m^{2} a_{1}^{2}\left\{G_{21} m+\left(-G_{02}+m\right) Q^{\prime \prime}(0)+m\left(G_{02}(9+m)-2 m(17+2 m)\right) a_{1}^{2}+4\left(G_{02}\right.\right. \\
& \left.\left.-3 m) m a_{1} a_{2}\right\} c_{1}^{2} c_{2}+12\left(G_{02}-2 m\right) m^{4} a_{1}^{4} c_{2}^{2}-24 m^{5} a_{1}^{4} c_{1} c_{3}\right] e_{n}^{8}+O\left(e_{n}^{9}\right) .
\end{aligned}
$$

The above asymptotic error constant (25) reveals that the proposed scheme (4) reaches at optimal eighth-order convergence by using only four functional evaluations (viz. $f\left(x_{n}\right) f^{\prime}\left(x_{n}\right) f\left(y_{n}\right)$ and $f\left(z_{n}\right)$ ) per iteration. This completes the proof.

Remark 1 It is important to note that the weight functions $Q$ and $G$ play a significant role in the construction of desired convergence order of the proposed scheme. However, only terms namely, $Q^{\prime \prime}(0), Q^{(3)}(0), Q^{(4)}(0), G_{02}, G_{21}$ and $G_{40}$ are involved in the asymptotic error constant term (25). On the other hand, $Q^{(5)}(0), G_{21}, G_{12}, G_{03}, G_{31}, G_{22}, G_{13}$ and $G_{04}$ do not affect the asymptotic error constant term (25). So, we can consider them as dummy parameters. However, we can't leave them in the beginning.

\subsection{Special cases of the proposed family}

In this section, we will discuss some special cases of our proposed class (4) by assigning different weight functions $Q$ and $G$. 
1. Let us consider the following weight functions which are chosen directly from the proposed Theorem 1 . Then, we get a new optimal class of order eight as follows:

$$
\begin{gathered}
y_{n}=x_{n}-m \frac{f\left(x_{n}\right)}{f^{\prime}\left(x_{n}\right)}, \\
z_{n}=y_{n}-\frac{f\left(x_{n}\right)}{f^{\prime}\left(x_{n}\right)} u_{n}\left[m+2 h_{n} m a_{1}+\frac{1}{2} h_{n}^{2} Q^{\prime \prime}(0)+\frac{1}{3 !} h_{n}^{3} Q^{(3)}(0)+\frac{1}{4 !} h_{n}^{4} Q^{(4)}(0)+\frac{1}{5 !} h_{n}^{5} Q^{(5)}(0)\right], \\
x_{n+1}=z_{n}-\frac{f\left(x_{n}\right)}{f^{\prime}\left(x_{n}\right)} u_{n} t_{n}\left[m+2 m a_{1} h_{n}+m t_{n}+\frac{1}{2 !}\left(\left(Q^{\prime \prime}(0)+2 m a_{1}^{2}\right) h_{n}^{2}+8 m a_{1} h_{n} t_{n}+G_{02} t_{n}^{2}\right)\right. \\
+\frac{1}{3 !}\left\{\left(Q^{(3)}(0)+6 Q^{\prime \prime}(0) a_{1}-24 m a_{1}^{3}-12 m a_{1}^{2} a_{2}\right) h_{n}^{3}+3 G_{21} h_{n}^{2} t_{n}+3 G_{12} h_{n} t_{n}^{2}+G_{03} t_{n}^{3}\right\} \\
\left.+\frac{1}{4 !}\left(G_{40} h_{n}^{4}+4 G_{31} h_{n}^{3} t_{n}+6 G_{22} h_{n}^{2} t_{n}^{2}+4 G_{13} h_{n} t_{n}^{3}+G_{04} t_{n}^{4}\right)\right],
\end{gathered}
$$

where $a_{1}$ and $a_{2}$ nonzero complex parameters, $Q^{\prime \prime}(0), Q^{(3)}(0), Q^{(4)}(0), Q^{(5)}(0), G_{02}, G_{12}, G_{21}, G_{03}, G_{40}, G_{31}, G_{22}, G_{13}$ and $G_{04}$ are free parameters.

Sub cases of the given scheme (26):

(a) For $Q^{\prime \prime}(0)=4 a_{1}^{2} m+2 m a_{1} a_{2}, Q^{(3)}(0)=Q^{(4)}(0)=Q^{(5)}(0)=G_{12}=G_{21}=G_{03}=G_{40}=G_{31}=G_{22}=G_{13}=$ $G_{04}=0$, in expression (26), we have

$$
\begin{aligned}
y_{n} & =x_{n}-m \frac{f\left(x_{n}\right)}{f^{\prime}\left(x_{n}\right)}, \\
z_{n} & =y_{n}-\frac{f\left(x_{n}\right)}{f^{\prime}\left(x_{n}\right)} u_{n}\left[m+2 h_{n} m a_{1}+\frac{1}{2} h_{n}^{2}\left(4 m a_{1}^{2}+2 m a_{1} a_{2}\right)\right], \\
x_{n+1} & =z_{n}-\frac{f\left(x_{n}\right)}{f^{\prime}\left(x_{n}\right)} u_{n} t_{n}\left[m+m t_{n}+\frac{G_{02} t_{n}^{2}}{2}+3 m a_{1}^{2} h_{n}^{2}+m a_{1} h_{n}\left(2+4 t_{n}+a_{2} h_{n}\right)\right] .
\end{aligned}
$$

(b) Let us consider $Q^{\prime \prime}(0)=Q^{(3)}(0)=Q^{(4)}(0)=Q^{(5)}(0)=G_{02}=G_{12}=G_{21}=G_{03}=G_{31}=G_{22}=G_{13}=G_{04}=$ 0 , in expression (26), we obtain

$$
\begin{aligned}
y_{n} & =x_{n}-m \frac{f\left(x_{n}\right)}{f^{\prime}\left(x_{n}\right)}, \\
z_{n} & =y_{n}-\frac{f\left(x_{n}\right)}{f^{\prime}\left(x_{n}\right)} u_{n}\left[m+2 h_{n} m a_{1}\right], \\
x_{n+1} & =z_{n}-\frac{f\left(x_{n}\right)}{f^{\prime}\left(x_{n}\right)} u_{n} t_{n}\left[m+m t_{n}+2 h_{n} m\left(1+2 t_{n}\right) a_{1}-4 h_{n}^{3} m a_{1}^{3}+h_{n}^{2} m a_{1}^{2}\left(1-2 h_{n} a_{2}\right)+\frac{G_{40} h_{n}^{4}}{24}\right] .
\end{aligned}
$$

2. Another choice of weight functions gives the following family of eighth-order methods

$$
\begin{aligned}
y_{n} & =x_{n}-m \frac{f\left(x_{n}\right)}{f^{\prime}\left(x_{n}\right)}, \\
z_{n} & =y_{n}-\frac{f\left(x_{n}\right)}{f^{\prime}\left(x_{n}\right)} u_{n}\left[m+2 h_{n} m a_{1}+\frac{1}{2} h_{n}^{2}\left(4 m a_{1}^{2}+2 m a_{1} a_{2}\right)\right], \\
x_{n+1} & =z_{n}-\frac{f\left(x_{n}\right)}{f^{\prime}\left(x_{n}\right)} u_{n} t_{n}\left[\frac{m\left(1+2 t_{n}+3 h^{2} a_{1}^{2}+h_{n} a_{1}\left(2+6 t_{n}+h_{n} a_{2}\right)\right)}{1+t_{n}}\right],
\end{aligned}
$$

where $a_{1}$ and $a_{2}$ non zero complex parameters.

3. By considering one more weight functions of the following form, we will obtain one more optimal family of eighth-order iteration function

$$
\begin{aligned}
y_{n} & =x_{n}-m \frac{f\left(x_{n}\right)}{f^{\prime}\left(x_{n}\right)}, \\
z_{n} & =y_{n}-\frac{f\left(x_{n}\right)}{f^{\prime}\left(x_{n}\right)} u_{n}\left[m+2 h_{n} m a_{1}+\frac{1}{2} h_{n}^{2}\left(4 m a_{1}^{2}+2 m a_{1} a_{2}\right)\right], \\
x_{n+1} & =z_{n}-\frac{f\left(x_{n}\right)}{f^{\prime}\left(x_{n}\right)} u_{n} t_{n}\left[-1+m+2 h_{n} m a_{1}+\frac{1+4 m a_{1} h_{n} t_{n}}{1-m t_{n}}+h_{n}^{2} m a_{1}\left(3 a_{1}+a_{2}\right)\right],
\end{aligned}
$$


where $a_{1}$ and $a_{2}$ non zero complex parameters.

4. We will get another optimal family of eighth-order methods with the following choice of weight functions

$$
\begin{aligned}
y_{n} & =x_{n}-m \frac{f\left(x_{n}\right)}{f^{\prime}\left(x_{n}\right)}, \\
z_{n} & =y_{n}-\frac{f\left(x_{n}\right)}{f^{\prime}\left(x_{n}\right)} u_{n}\left[\frac{m\left(h_{n}-4 m a_{1}-8 h_{n} m a_{1}^{2}\right)}{h_{n}-4 m a_{1}}\right], \\
x_{n+1} & =z_{n}-m \frac{f\left(x_{n}\right)}{f^{\prime}\left(x_{n}\right)} u_{n} t_{n}\left[1+t_{n}+2 h_{n} a_{1}\left(1+2 t_{n}+\frac{h_{n}\left(1+2 m a_{1}^{2}\right)^{2}}{-h_{n}+4 m a_{1}\left\{1+2 a_{1}\left(m a_{1}\left(1+4 h_{n} a_{1}+2 h_{n} a_{2}\right)-h_{n}\right)\right\}}\right)\right],
\end{aligned}
$$

where $a_{1}$ and $a_{2}$ non zero complex parameters.

In the similar fashion by choosing arbitrary weight functions of $Q\left(h_{n}\right)$ and $G\left(h_{n}, t_{n}\right)$ (provided the conditions on them in the above Theorem 1 should be satisfied), we can obtain several new optimal methods of eighth-order for multiple zeros.

\section{Numerical experiments}

In this section, we will check the efficiency and convergence behavior of our proposed iteration functions namely, expression (27) for $\left(a_{1}=1, a_{2}=-2, G_{02}=2 m\right)$ and $\left(a_{1}=1, a_{2}=1, G_{02}=0\right)$ and expression (29) for $\left(a_{1}=1, a_{2}=1\right)$ denoted by OM1, OM2 and OM3, respectively. In this regard, we consider a total number of four test problems: first one is a real life problem; second one is from the linear algebra; third one is a standard test problem; fourth one again from real life but for simple zeros, which are mentioned in Examples 1 to 5.

Unfortunately, we don't have any optimal eighth-order iteration function for multiple zeros for the comparison. So, we consider the highest-order methods of order six (multiple zeros) for comparison which is available in the literature. Now, we compare our proposed methods with family of two-point sixth-order method given by Geum et al. in [15], out of them we choose the case $4 \mathrm{c}$, which is given by:

$$
\begin{aligned}
y_{n} & =x_{n}-m \frac{f\left(x_{n}\right)}{f^{\prime}\left(x_{n}\right)}, m>1 \\
x_{n+1} & =y_{n}-\left[\frac{m+a_{1} u_{n}}{1+b_{1} u_{n}+b_{2} u_{n}^{2}} \times \frac{1}{1+c_{1} s_{n}}\right] \frac{f\left(y_{n}\right)}{f^{\prime}\left(y_{n}\right)},
\end{aligned}
$$

where $a_{1}=\frac{2 m\left(4 m^{4}-16 m^{3}+31 m^{2}-30 m+13\right)}{(m-1)\left(4 m^{2}-8 m+7\right)}, b_{1}=\frac{4\left(2 m^{2}-4 m+3\right)}{(m-1)\left(4 m^{2}-8 m+7\right)}$ and $b_{2}=-\frac{4 m^{2}-8 m+3}{4 m^{2}-8 m+7}$ and $c_{1}=2(m-1)$, called GKN1.

Finally, we compare them with the non optimal family of sixth-order methods based on weight function approach presented by the same authors Geum et al. [16], out of them we consider the case 5YD, which is defined as follows:

$$
\begin{aligned}
y_{n} & =x_{n}-m \frac{f\left(x_{n}\right)}{f^{\prime}\left(x_{n}\right)}, m \geq 1, \\
w_{n} & =x_{n}-m\left[\frac{\left(u_{n}-2\right)\left(2 u_{n}-1\right)}{\left(u_{n}-1\right)\left(5 u_{n}-2\right)}\right] \frac{f\left(x_{n}\right)}{f^{\prime}\left(x_{n}\right)}, \\
x_{n+1} & =x_{n}-m\left[\frac{\left(u_{n}-2\right)\left(2 u_{n}-1\right)}{\left(5 u_{n}-2\right)\left(u_{n}+v_{n}-1\right)}\right] \frac{f\left(x_{n}\right)}{f^{\prime}\left(x_{n}\right)},
\end{aligned}
$$

denoted by GKN2.

In this section, the comparison of performance of the proposed and existing methods is made by means of two different tools: dynamical phase portraits of the stability behavior of all the methods on the complex plane, that allows us to know how wide is the set of initial estimations that lead us to the sought roots and the usual numerical test based on high-precision calculations on few initial estimations. Both of them give us complementary information that helps us to fully understand the numerical performance of the iterative schemes and to establish founded conclusions.

Regarding the stability comparison, we use the routines presented in [7] for plotting the dynamical planes corresponding to each method (OM1, OM2, OM3, GKN1 and GKN2) for each one of the nonlinear functions studied. In them, a mesh of $800 \times 800$ points is defined, as each point of the mesh is an initial estimation for the analyzed method on the specific problem. 
If the method reaches (closer than $10^{-3}$ ) one of the roots (multiple or not) in less than 200 iterations, then this point is painted in (orange, red, green,...) color; if the iterate diverges (tends to the infinity), the point is painted blue color; in other cases, the point is painted black.

In the numerical tests presented in Tables 1 to 5, we have compared our methods with the known ones on the basis of approximated zeros, residual error of the involved functions, difference between the two consecutive iterations, asymptotic error constants. In Tables $1-5$, we display the number of iteration indices $(n)$, approximated zeros $\left(x_{n}\right)$, absolute residual error of the corresponding function $\left(\left|f\left(x_{n}\right)\right|\right)$, error in the consecutive iterations $\left|x_{n+1}-x_{n}\right|$, computational order of convergence $\rho=\frac{\log \left|\left(x_{n+1}-x_{n}\right) /\left(x_{n}-x_{n-1}\right)\right|}{\log \left|\left(x_{n}-x_{n-1}\right) /\left(x_{n-1}-x_{n-2}\right)\right|}, n \geq 2$, (the details of this formula can be seen in Cordero and Torregrosa [19]), $\left|\frac{x_{n+1}-x_{n}}{\left(x_{n}-x_{n-1}\right)^{p}}\right|$ (where $p$ is either 6 or 8 corresponding to the considered iteration function), the estimation of asymptotic error constant $\eta \approx \lim _{n \rightarrow \infty}\left|\frac{x_{n+1}-x_{n}}{\left(x_{n}-x_{n-1}\right)^{p}}\right|$ at the last iteration. We make our calculations with several number of significant digits (minimum 3000 significant digits) to minimize the roundoff error.

As we mentioned in the above paragraph, we calculate the values of all the constants and functional residuals up to several number of significant digits but we display the value of $x_{n}$ up to 25 significant digits. In addition, we also display $\left|\frac{x_{n+1}-x_{n}}{\left(x_{n}-x_{n-1}\right)^{p}}\right|$ and $\eta$ up to 10 significant digits. Moreover, absolute residual error in the function $\left|f\left(x_{n}\right)\right|$ and error in the consecutive iterations $\left|x_{n+1}-x_{n}\right|$ are displayed up to 2 significant digits with exponent power which are mentioned in Tables $1-5$. Finally, computational order of convergence is up to 5 significant digits. Furthermore, the approximated zeros up to 25 significant digits are also displayed in the Examples 1-5 although minimum 3000 significant digits are available with us.

For these numerical tests, all computations have been performed using the programming package Mathematica 11 with multiple precision arithmetic. Further, the meaning of $a( \pm b)$ is $a \times 10^{( \pm b)}$ in Tables $1-5$.

Example 1 Van der Waals equation of state

$$
\left(P+\frac{a_{1} n^{2}}{V^{2}}\right)\left(V-n a_{2}\right)=n R T,
$$

explains the behavior of a real gas by introducing in the ideal gas equations two parameters, $\alpha_{1}$ and $\alpha_{2}$, specific for each gas. The determination of the volume $V$ of the gas in terms of the remaining parameters requires the solution of a nonlinear equation in $\mathrm{V}$

$$
P V^{3}-\left(n a_{2} P+n R T\right) V^{2}+\alpha_{1} n^{2} V-\alpha_{1} \alpha_{2} n^{2}=0 .
$$

Given the constants $\alpha_{1}$ and $\alpha_{2}$ of a particular gas, one can find values for $n, P$ and $T$, such that this equation has a three simple roots. By using the particular values, we obtain the following nonlinear function

$$
f_{1}(x)=x^{3}-5.22 x^{2}+9.0825 x-5.2675
$$

have three zeros and out of them one is a multiple zero $\alpha=1.75$ of multiplicity of order two and other one simple zero $\alpha=1.72$. However, our desired root is $\alpha=1.75$.

Regarding the dynamical behavior, it can be observed in Figures 1 and 2 that the only basin of attraction is that of the multiple root (that is, the set of initial points converging to it fills all the plotted region $[-3,3] \times[-3,3]$ of the complex plane), plotted in orange in the figures; although in general, convergence to other roots, divergence or even convergence to other fixed points that are not roots of the nonlinear function (known as strange fixed points), can appear. In fact, it can be observed in Figure $2 b$ that black regions appear in the center of slow-convergence regions. On the other hand, the rest of schemes have a very stable performance, with global convergence to the multiple root.

On the other hand, the numerical results presented in Table 1 show that, with the same initial estimation in the orange stable area of all the methods, those having better performance in terms of precision in the calculation of the root are the new methods OM1, OM2 and OM3. The numerical estimation of the order of convergence coincide with the theoretical one in all cases. 


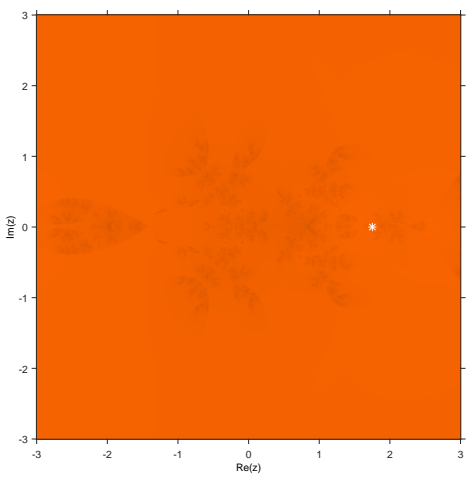

(a) OM1

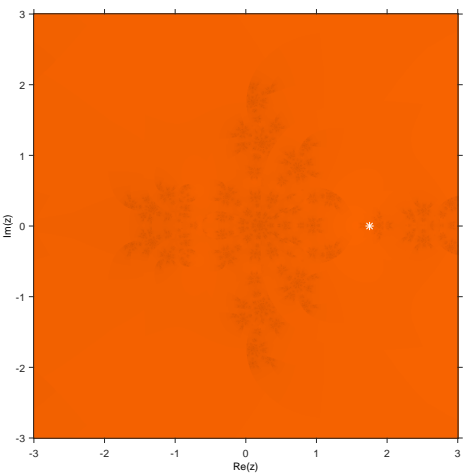

(b) OM2

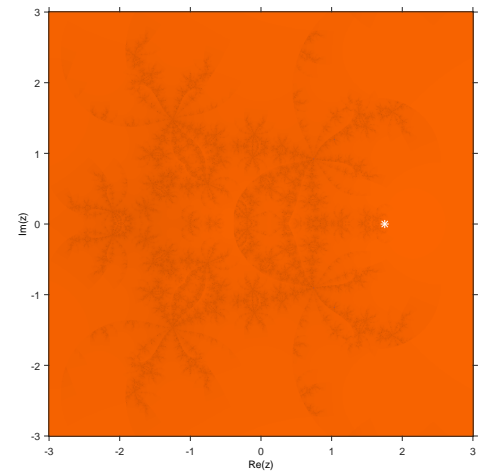

(c) OM3

Fig. 1: Dynamical plane of proposed methods on $f_{1}(x)$

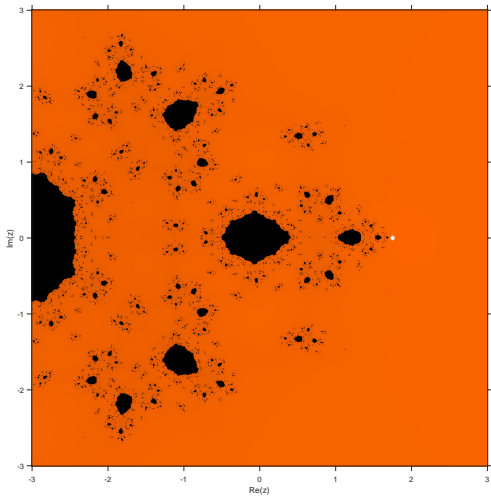

(a) GKN1

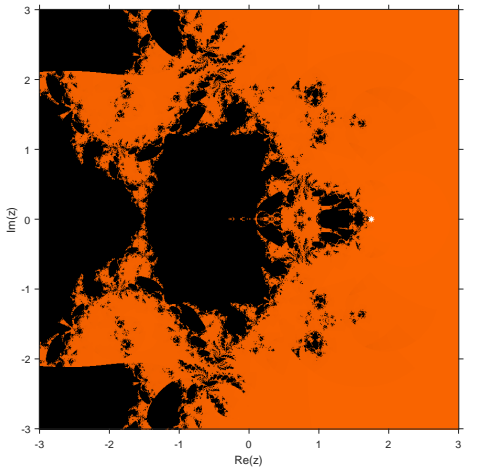

(b) GKN2

Fig. 2: Dynamical plane of existing methods on $f_{1}(x)$

Table 1: Convergence behavior of different iterative methods on the test function $f_{1}(x)$

\begin{tabular}{|c|c|c|c|c|c|c|c|}
\hline Cases & $n$ & $x_{n}$ & $\left|f\left(x_{n}\right)\right|$ & $\left|x_{n+1}-x_{n}\right|$ & $\rho$ & $\frac{x_{n+1}-x_{n}}{\left(x_{n}-x_{n-1}\right)^{p}}$ & $\eta$ \\
\hline \multirow{4}{*}{$G K N 1$} & 0 & 1.8 & $2.0(-4)$ & $4.9(-2)$ & & & \multirow{4}{*}{$4.831716596(+7)$} \\
\hline & 1 & 1.750953332157375836273933 & $2.2(-8)$ & $9.5(-4)$ & & $6.848369523(+4)$ & \\
\hline & 2 & 1.750000000027291304626704 & $2.2(-23)$ & $2.7(-11)$ & & $3.635456190(+7)$ & \\
\hline & 3 & 1.750000000000000000000000 & $1.2(-113)$ & $2.0(-56)$ & 5.9836 & $4.831716596(+7)$ & \\
\hline \multirow{4}{*}{$G K N 2$} & 0 & 1.8 & $2.0(-4)$ & $5.0(-2)$ & & & \multirow{4}{*}{$3.215020576(+6)$} \\
\hline & 1 & 1.750388172793891559741273 & $4.6(-9)$ & $3.9(-4)$ & & $2.603237303(+4)$ & \\
\hline & 2 & 1.750000000000010343224637 & $3.2(-30)$ & $1.0(-14)$ & & $3.023468138(+6)$ & \\
\hline & 3 & 1.750000000000000000000000 & $4.6(-157)$ & $3.9(-78)$ & 5.9975 & $3.215020576(+6)$ & \\
\hline \multirow{4}{*}{$O M 1$} & 0 & 1.8 & $2.0(-4)$ & $5.0(-2)$ & & & \multirow{4}{*}{$3.750857339(+9)$} \\
\hline & 1 & 1.750164271144510471766995 & $8.1(-10)$ & $1.6(-4)$ & & $4.317524084(+6)$ & \\
\hline & 2 & 1.750000000000000000001923 & $1.1(-43)$ & $1.9(-21)$ & & $3.626854132(+9)$ & \\
\hline & 3 & 1.750000000000000000000000 & $1.5(-314)$ & $7.0(-157)$ & 7.9991 & $3.750857339(+9)$ & \\
\hline \multirow{4}{*}{$O M 2$} & 0 & 1.8 & $2.0(-4)$ & $5.0(-2)$ & & & \multirow{4}{*}{$8.479616770(+10)$} \\
\hline & 1 & 1.750453249826572295479110 & $6.3(-9)$ & $4.5(-4)$ & & $1.248004799(+7)$ & \\
\hline & 2 & 1.750000000000000131100951 & $5.2(-34)$ & $1.3(-16)$ & & $7.360455121(+10)$ & \\
\hline & 3 & 1.750000000000000000000000 & $1.6(-234)$ & $7.4(-117)$ & 7.9951 & $8.479616770(+10)$ & \\
\hline \multirow{4}{*}{$O M 3$} & 0 & 1.8 & $2.0(-4)$ & $4.9(-2)$ & & & \multirow{4}{*}{$1.462834362(+11)$} \\
\hline & 1 & 1.750570071950781672220702 & $9.9(-9)$ & $5.7(-4)$ & & $1.599594295(+7)$ & \\
\hline & 2 & 1.750000000000001356336629 & $5.5(-32)$ & $1.4(-15)$ & & $1.215990289(+11)$ & \\
\hline & 3 & 1.750000000000000000000000 & $8.4(-218)$ & $1.7(-108)$ & 7.9931 & $1.462834362(+11)$ & \\
\hline
\end{tabular}


Example 2 Consider the following $8 \times 8$ matrix

$$
A=\left[\begin{array}{cccccccc}
-12 & -12 & 36 & -12 & 0 & 0 & 12 & 8 \\
148 & 129 & -397 & 147 & -12 & 6 & -109 & -74 \\
72 & 62 & -186 & 66 & -8 & 4 & -54 & -36 \\
-32 & -24 & 88 & -36 & 0 & 0 & 24 & 16 \\
20 & 13 & -45 & 19 & 8 & 6 & -13 & -10 \\
120 & 98 & -330 & 134 & -8 & 24 & -90 & -60 \\
-132 & -109 & 333 & -115 & 12 & -6 & 105 & 66 \\
0 & 0 & 0 & 0 & 0 & 0 & 0 & 4
\end{array}\right]
$$

The corresponding characteristic polynomial of this matrix is as follows:

$$
f_{2}(x)=(x-4)^{3}(x+4)(x-8)(x-20)(x-12)(x+12) .
$$

It's characteristic equation has one multiple root at $x=4$ of multiplicity three.

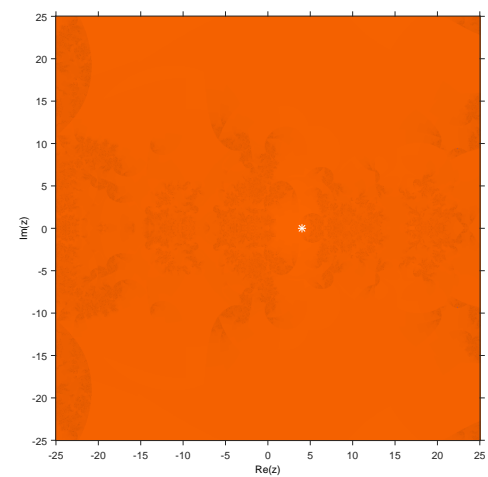

(a) OM1

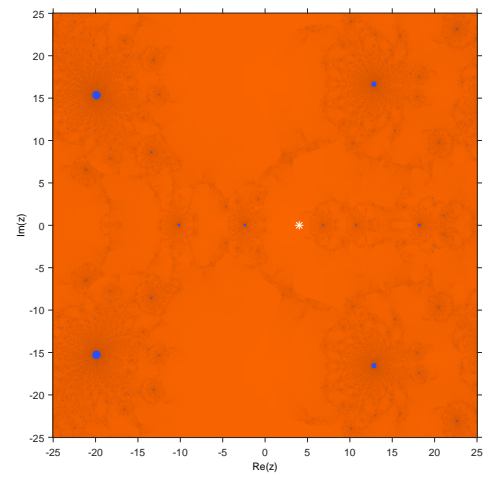

(b) $\mathrm{OM} 2$

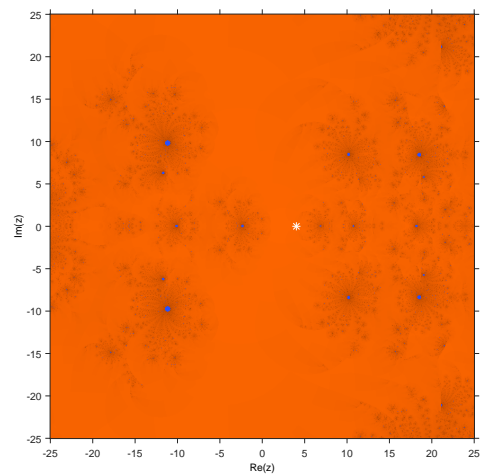

(c) OM3

Fig. 3: Dynamical plane of proposed methods on $f_{2}(x)$

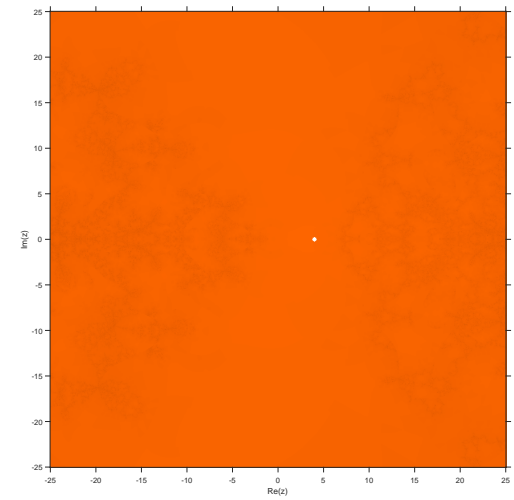

(a) GKN1

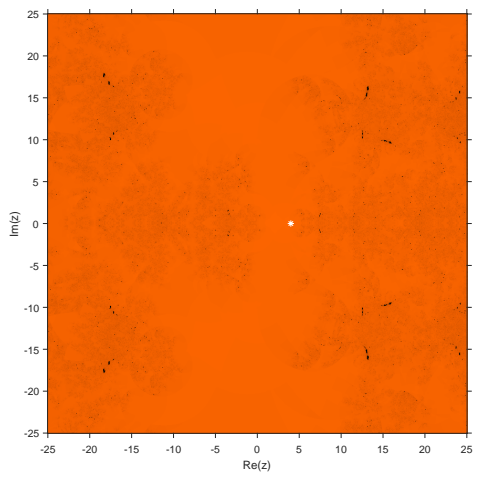

(b) GKN2

Fig. 4: Dynamical plane of existing methods on $f_{2}(x)$

It can be observed in Figures $3 b$ and $3 c$ that several blue areas appear; they correspond to the basin of attraction of infinity. This means that the method diverges if the initial estimation used is inside one of these blue regions. The schemes that are 
free of divergence are OM1, GKN1 and GKN2, as can be observed in Figures 3a, 4a and 4b, where global convergence to the multiple root is observed. Indeed, the numerical tests made with this nonlinear function show that the best results in terms of precision and estimation of the order of convergence is again OM1 but also OM2 and OM3 show better results of the known methods of sixth-order of convergence.

Table 2: Convergence behavior of different iterative methods on the test function $f_{2}(x)$

\begin{tabular}{|c|c|c|c|c|c|c|c|}
\hline Cases & $n$ & $x_{n}$ & $\left|f\left(x_{n}\right)\right|$ & $\left|x_{n+1}-x_{n}\right|$ & $\rho$ & $\frac{x_{n+1}-x_{n}}{\left(x_{n}-x_{n-1}\right)^{p}}$ & $\eta$ \\
\hline \multirow{4}{*}{$G K N 1$} & 0 & 3.8 & $5.5(+2)$ & $2.0(-1)$ & & & \multirow{4}{*}{$2.641111201(-4)$} \\
\hline & 1 & 3.999999989382956352637933 & $7.8(-20)$ & $1.1(-8)$ & & $1.658913598(-4)$ & \\
\hline & 2 & 4.000000000000000000000000 & $3.5(-150)$ & $3.8(-52)$ & & $2.641111134(-4)$ & \\
\hline & 3 & 4.000000000000000000000000 & $3.0(-932)$ & $7.7(-132)$ & 6.0000 & $2.641111201(-4)$ & \\
\hline \multirow{4}{*}{$G K N 2$} & 0 & 3.8 & $5.5(+2)$ & $2.0(-1)$ & & & \multirow{4}{*}{$3.857082791(-4)$} \\
\hline & 1 & 3.999999998020003959588340 & $5.1(-22)$ & $2.0(-9)$ & & $3.093743997(-4)$ & \\
\hline & 2 & 4.000000000000000000000000 & $8.2(-166)$ & $2.3(-57)$ & & $3.857082782(-4)$ & \\
\hline & 3 & 4.000000000000000000000000 & $1.5(-1028)$ & $6.1(-345)$ & 6.0000 & $3.857082791(-4)$ & \\
\hline \multirow{4}{*}{$O M 1$} & 0 & 3.8 & $5.5(+2)$ & $2.0(-1)$ & & & \multirow{4}{*}{$1.181881705(-6)$} \\
\hline & 1 & 3.999999999997811832679154 & $6.9(-31)$ & $2.2(-12)$ & & $8.547528598(-7)$ & \\
\hline & 2 & 4.000000000000000000000000 & $1.6(-293)$ & $6.2(-100)$ & & $1.181881705(-6)$ & \\
\hline & 3 & 4.000000000000000000000000 & $1.2(-2394)$ & $2.6(-800)$ & 8.0000 & $1.181881705(-6)$ & \\
\hline \multirow{4}{*}{$O M 2$} & 0 & 3.8 & $5.5(+2)$ & $2.0(-1)$ & & & \multirow{4}{*}{$1.459992426(-5)$} \\
\hline & 1 & 3.999999999977418636628025 & $7.5(-28)$ & $2.3(-11)$ & & $8.820845075(-6)$ & \\
\hline & 2 & 4.000000000000000000000000 & $6.3(-266)$ & $9.9(-91)$ & & $1.459992426(-5)$ & \\
\hline & 3 & 4.000000000000000000000000 & $1.5(-2170)$ & $1.3(-725)$ & 8.0000 & $1.459992426(-5)$ & \\
\hline \multirow{4}{*}{$O M 3$} & 0 & 3.8 & $5.5(+2)$ & $2.0(-1)$ & & & \multirow{4}{*}{$2.437715050(-5)$} \\
\hline & 1 & 3.999999999962682937655212 & $3.4(-27)$ & $3.7(-11)$ & & $1.457697750(-5)$ & \\
\hline & 2 & 4.000000000000000000000000 & $5.5(-260)$ & $9.2(-89)$ & & $2.437715050(-5)$ & \\
\hline & 3 & 4.000000000000000000000000 & $1.2(-2122)$ & $1.2(-709)$ & 8.0000 & $2.437715050(-5)$ & \\
\hline
\end{tabular}

Example 3 Let us pick a standard nonlinear test function from [4], which is given as follows:

$$
f_{3}(x)=\left((x-1)^{3}-1\right)^{50},
$$

The above function has a multiple root at $x=2$ of multiplicity 50 .

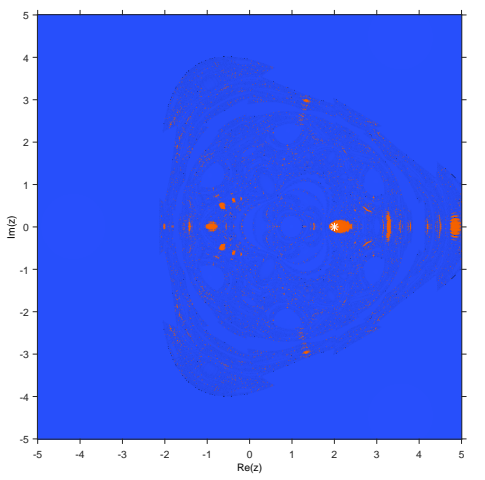

(a) $\mathrm{OM} 1$

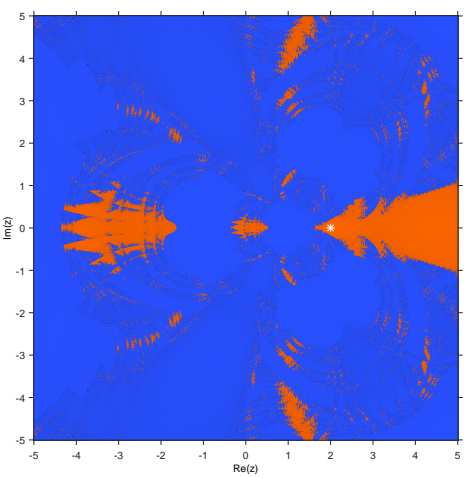

(b) OM2

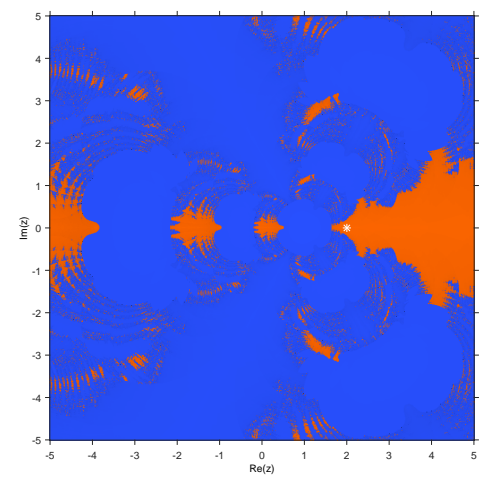

(c) $\mathrm{OM} 3$

Fig. 5: Dynamical plane of proposed methods on $f_{3}(x)$

In Figures 5 and 6 it is observed that the basin of attraction of the multiple root (in orange in the figure) is bigger at the right size of the region $[-5,5] \times[-5,5]$. In fact, it is very good for real values greater than the root for all the methods 


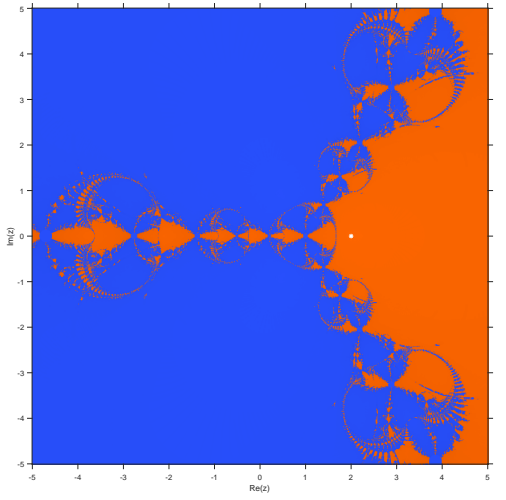

(a) GKN1

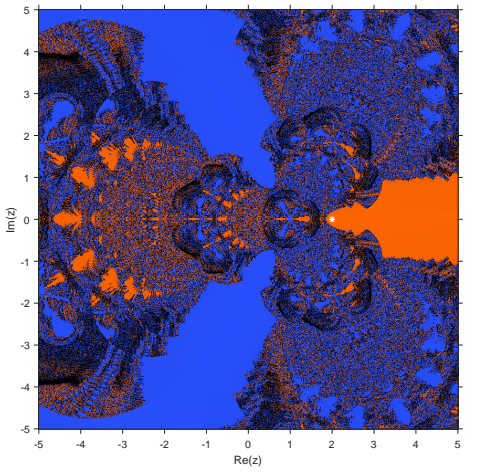

(b) GKN2

Fig. 6: Dynamical plane of existing methods on $f_{3}(x)$

(except OM1), in particular for GKN1. In all the schemes the blue region of divergence is also very extensive. This can also be observed in the numerical test showed in Table 3, where the results are very good for all the methods (the initial estimation is close to the root and greater than it), being lower the residuals at the new methods. This is justified, as in all cases, for the higher order of convergence of the proposed methods.

Table 3: Convergence behavior of different iterative methods on the test function $f_{3}(x)$.

\begin{tabular}{|c|c|c|c|c|c|c|c|}
\hline Cases & $n$ & $x_{n}$ & $\left|f\left(x_{n}\right)\right|$ & $\left|x_{n+1}-x_{n}\right|$ & $\rho$ & $\frac{x_{n+1}-x_{n}}{\left(x_{n}-x_{n-1}\right)^{p}}$ & $\eta$ \\
\hline \multirow{4}{*}{$G K N 1$} & 0 & 2.1 & $9.8(-25)$ & $1.0(-1)$ & & & \multirow{4}{*}{$3.361366099(+2)$} \\
\hline & 1 & 2.000017940282672234796082 & $3.5(-214)$ & $1.8(-5)$ & & $1.795960603(+1)$ & \\
\hline & 2 & 2.000000000000000000000000 & $1.9(-1274)$ & $1.1(-26)$ & & $3.354982324(+2)$ & \\
\hline & 3 & 2.000000000000000000000000 & $6.0(-7636)$ & $6.6(-154)$ & 6.0000 & $3.361366099(+2)$ & \\
\hline \multirow{4}{*}{$G K N 2$} & 0 & 2.1 & $9.8(-25)$ & $1.0(-1)$ & & & \multirow{4}{*}{$2.777777778(-1)$} \\
\hline & 1 & 2.000000200989638086020762 & $1.0(-311)$ & $2.0(-7)$ & & $2.009920619(-1)$ & \\
\hline & 2 & 2.000000000000000000000000 & $9.8(-2014)$ & $1.8(-41)$ & & $2.777775861(-1)$ & \\
\hline & 3 & 2.000000000000000000000000 & $7.3(-12226)$ & $1.0(-245)$ & 6.0000 & $2.777777778(-1)$ & \\
\hline \multirow{4}{*}{$O M 1$} & 0 & 2.1 & $9.8(-25)$ & $1.0(-1)$ & & & \multirow{4}{*}{1.555555556} \\
\hline & 1 & 2.000000010138026577344089 & $1.4(-376)$ & $1.0(-8)$ & & 1.013803480 & \\
\hline & 2 & 2.000000000000000000000000 & $6.8(-3165)$ & $1.7(-64)$ & & 1.555555492 & \\
\hline & 3 & 2.000000000000000000000000 & $1.8(-25471)$ & $1.3(-510)$ & 8.0000 & 1.555555556 & \\
\hline \multirow{4}{*}{$O M 2$} & 0 & 2.1 & $9.8(-25)$ & $1.0(-1)$ & & & \multirow{4}{*}{$1.238518519(+2)$} \\
\hline & 1 & 2.000000465433503138049861 & $1.8(-293)$ & $4.7(-7)$ & & $4.654508338(+1)$ & \\
\hline & 2 & 2.000000000000000000000000 & $4.4(-2405)$ & $2.7(-49)$ & & $1.238513513(+2)$ & \\
\hline & 3 & 2.000000000000000000000000 & $6.4(-19298)$ & $3.8(-387)$ & 8.0000 & $1.238518519(+2)$ & \\
\hline \multirow{4}{*}{$O M 3$} & 0 & 2.1 & $9.8(-25)$ & $1.0(-1)$ & & & \multirow{4}{*}{$2.269259259(+2)$} \\
\hline & 1 & 2.000000785189010712446522 & $4.0(-282)$ & $7.9(-7)$ & & $7.852383342(+1)$ & \\
\hline & 2 & 2.000000000000000000000000 & $4.4(-2301)$ & $3.3(-47)$ & & $2.269242109(+2)$ & \\
\hline & 3 & 2.000000000000000000000000 & $8.3(-18453)$ & $3.0(-370)$ & 8.0000 & $2.269259259(+2)$ & \\
\hline
\end{tabular}

Example 4 Fractional conversion in a chemical reactor:

Let us consider the following expression (for the details of this problem please see [18])

$$
f_{4}(x)=\frac{x}{1-x}-5 \log \left[\frac{0.4(1-x)}{0.4-0.5 x}\right]+4.45977 .
$$

In the above expression $x$ represents the fractional conversion of species A in a chemical reactor. Since, there is no physical meaning of above fractional conversion if $x$ is less than zero or greater than one. In this sense, $x$ is bounded in the region $0 \leq x \leq 1$. In addition, our required zero (that is simple) to this problem is $\alpha \approx 0.757396246253753879459641297929$. 
Moreover, it is interesting to note that the above expression is undefined in the region $0.8 \leq x \leq 1$ which is very close to our desired zero. Furthermore, there are some other properties to this function which make the solution more difficult. The derivative of the above expression is very close to zero in the region $0 \leq x \leq 0.5$ and there is an infeasible solution for $x=1.098$.

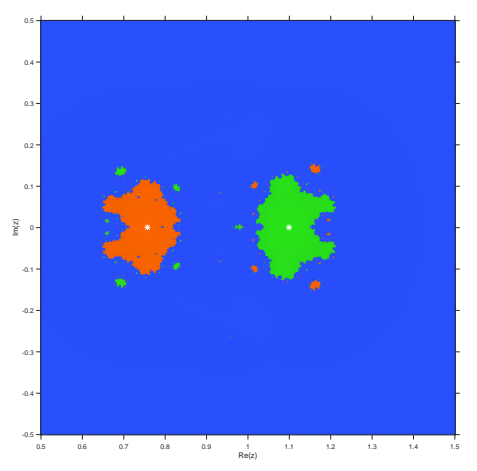

(a) $\mathrm{OM} 1$

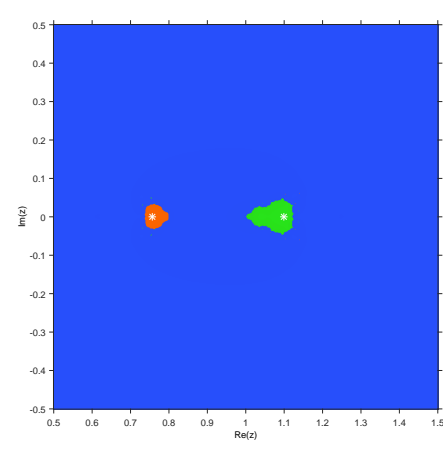

(b) $\mathrm{OM} 2$

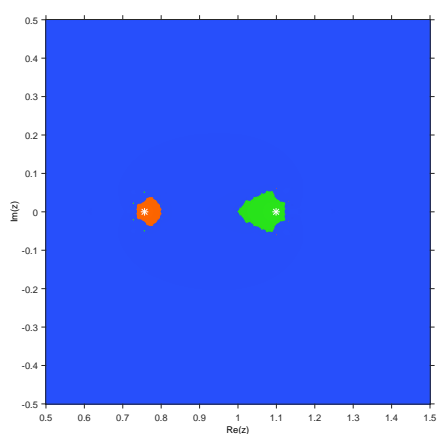

(c) $\mathrm{OM} 3$

Fig. 7: Dynamical plane of proposed methods on $f_{4}(x)$

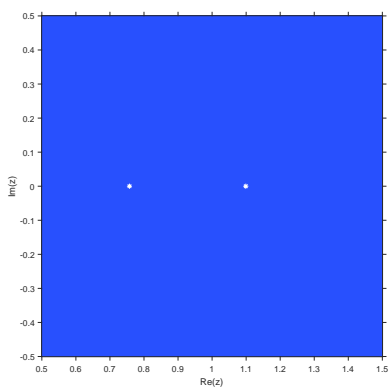

(a) GKN1

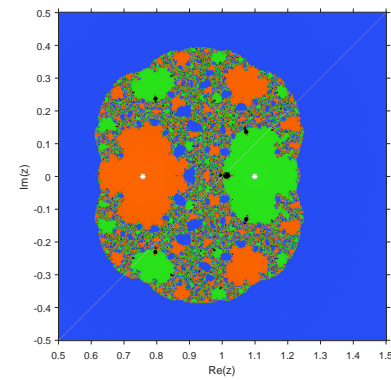

(b) GKN2

Fig. 8: Dynamical plane of existing methods on $f_{4}(x)$

In Figures 7 and 8 it is observed that the basin of attraction of the searched root (in orange in the figure) is quite small or does not exist in the most of the methods (except OM1 and GKN2, where it is bigger). Moreover, an almost symmetric colored area appears in some cases, that corresponds to the solution without physical sense, that is an attracting fixed point. All methods, except GKN1, get convergence to the multiple root for initial guesses close to it, although the basins of attraction are quite small in all cases, due to both are simple and the methods are specific for finding multiple ones. Regarding the numerical results appearing in Table 4, are compatible with the dynamical results and the order of the methods. In it, ${ }^{*}$ means that the method does not converge.

Example 5 Let us consider the following standard test function, which is given in Geum et al. [16]

$$
f_{5}(x)=(x-1)^{2}+\frac{1}{12}-\log \left[\frac{25}{12}-2 x+x^{2}\right] \text {. }
$$

Here, $\log z(z \in \mathbb{C})$ represents a principal analytic branch with $-\pi \leq \operatorname{Im}(\log z) \leq \pi$. The above function $f_{5}$ has multiple zeros $\xi=1 \pm i \frac{\sqrt{3}}{2}$ of multiplicity $m=2$.

Figures 9 and 10 show the dynamical planes of all the methods on $f_{5}$ in $[-2,2] \times[-2,2]$. It can be observed that only ones without black areas are OM1 and GKN1, being the last one the most stable. The unstable behavior is also present in Table 5 since $\rho$ does not reach the theoretical order of convergence. 
Table 4: Convergence behavior of different iterative methods on the test function $f_{4}(x)$

\begin{tabular}{|c|c|c|c|c|c|c|c|}
\hline Cases & $n$ & $x_{n}$ & $\left|f\left(x_{n}\right)\right|$ & $\left|x_{n+1}-x_{n}\right|$ & $\rho$ & $\frac{x_{n+1}-x_{n}}{\left(x_{n}-x_{n-1}\right)^{p}}$ & $\eta$ \\
\hline \multirow{4}{*}{$G K N 1$} & 0 & 0.76 & $*$ & $*$ & & & \multirow{4}{*}{ * } \\
\hline & 1 & & $*$ & $*$ & & $*$ & \\
\hline & 2 & & $*$ & $*$ & & $*$ & \\
\hline & 3 & & $*$ & $*$ & * & $*$ & \\
\hline \multirow{4}{*}{$G K N 2$} & 0 & 0.76 & $2.2(-1)$ & $2.6(-3)$ & & & \multirow{4}{*}{$5.257130496(+5)$} \\
\hline & 1 & 0.7573962460753336221899798 & $1.4(-8)$ & $1.8(-10)$ & & $5.725910242(+5)$ & \\
\hline & 2 & 0.7573962462537538794596413 & $1.4(-51)$ & $1.7(-53)$ & & $5.257130467(+5)$ & \\
\hline & 3 & 0.7573962462537538794596413 & $1.0(-309)$ & $1.3(-311)$ & 6.000 & $5.257130496(+5)$ & \\
\hline \multirow{4}{*}{$O M 1$} & 0 & 0.76 & $2.2(-1)$ & $2.6(-3)$ & & & \multirow{4}{*}{$1.146034267(+6)$} \\
\hline & 1 & 0.7573962462537572577085665 & $2.7(-13)$ & $3.4(-15)$ & & $1.599161718(+6)$ & \\
\hline & 2 & 0.7573962462537538794596413 & $1.6(-108)$ & $1.9(-110)$ & & $1.146034267(+6)$ & \\
\hline & 3 & 0.7573962462537538794596413 & $1.9(-870)$ & $2.3(-872)$ & 8.0000 & $1.146034267(+6)$ & \\
\hline \multirow{4}{*}{$O M 2$} & 0 & 0.76 & $2.2(-1)$ & $2.6(-3)$ & & & \multirow{4}{*}{$1.493537308(+10)$} \\
\hline & 1 & 0.7573962462850884293618686 & $2.5(-9)$ & $3.1(-11)$ & & $1.483283754(+10)$ & \\
\hline & 2 & 0.7573962462537538794596413 & $1.1(-72)$ & $1.4(-74)$ & & $1.493537308(+10)$ & \\
\hline & 3 & 0.7573962462537538794596413 & $1.6(-579)$ & $2.1(-581)$ & 8.0000 & $1.493537308(+10)$ & \\
\hline \multirow{4}{*}{$O M 3$} & 0 & 0.76 & $2.2(-1)$ & $2.6(-3)$ & & & \multirow{4}{*}{$3.013467463(+10)$} \\
\hline & 1 & 0.7573962463137703385994168 & $4.8(-9)$ & $6.0(-11)$ & & $2.840999693(+10)$ & \\
\hline & 2 & 0.7573962462537538794596413 & $4.0(-70)$ & $5.1(-72)$ & & $3.013467461(+10)$ & \\
\hline & 3 & 0.7573962462537538794596413 & $1.1(-558)$ & $1.3(-560)$ & 8.0000 & $3.013467463(+10)$ & \\
\hline
\end{tabular}

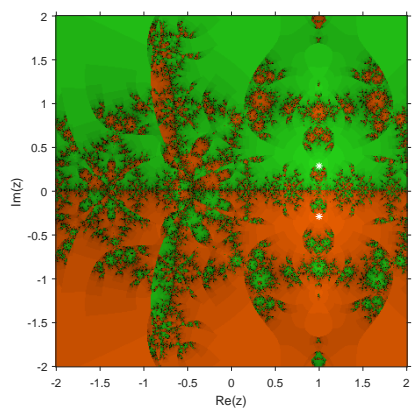

(a) OM1

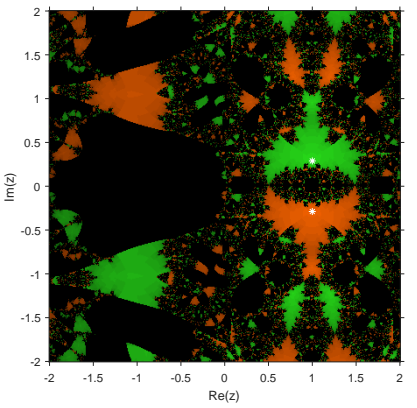

(b) $\mathrm{OM} 2$

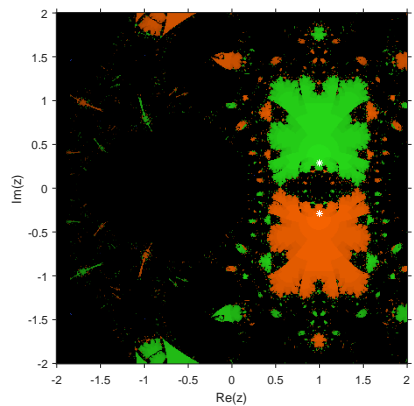

(c) OM3

Fig. 9: Dynamical plane of proposed methods on $f_{5}(x)$

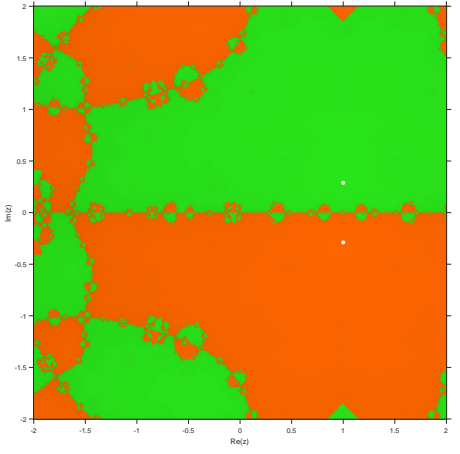

(a) GKN1

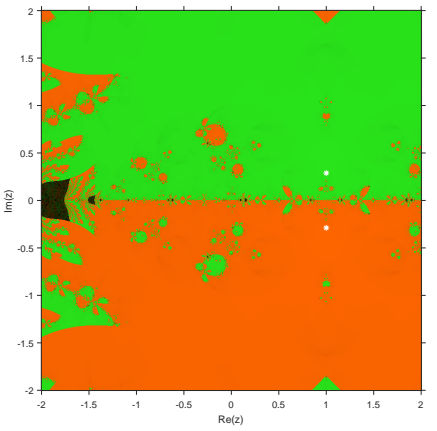

(b) GKN2

Fig. 10: Dynamical plane of existing methods on $f_{5}(x)$

Remark 2 From the computational Tables 1-5 we have observed that the smaller asymptotic error constant does not mean that the corresponding method converge faster than the other ones. For example, OM1 have smaller residual errors and smaller 
Table 5: Convergence behavior of different iterative methods on the test function $f_{5}(x)$.

\begin{tabular}{|c|c|c|c|c|c|c|c|}
\hline Cases & & $x_{n}$ & $\left|f\left(x_{n}\right)\right|$ & $\left|x_{n+1}-x_{n}\right|$ & $\rho$ & $\frac{x_{n+1}-x_{n}}{\left(x_{n}-x_{n-1}\right)^{p}}$ & $\eta$ \\
\hline \multirow{3}{*}{$G K N 1$} & 0 & $1.05-0.28 i$ & $4.2(-4)$ & $5.1(-2)$ & & & \multirow{3}{*}{$1.175789533(+42)$} \\
\hline & 1 & $1.0000029218954764616980048-0.2886638841214034969044654 i$ & $2.3(-11)$ & $1.2(-5)$ & & $6.809717133(+2)$ & \\
\hline & 2 & $1.0000000000000160702292310-0.2886751345947960530561430 i$ & $9.0(-29)$ & $2.3(-14)$ & & $9.434532257(+15)$ & \\
\hline \multirow{4}{*}{$G K N 2$} & 0 & $1.05-0.28 i$ & $4.2(-4)$ & $5.1(-2)$ & & & \multirow{4}{*}{$1.469994999(+25)$} \\
\hline & 1 & $0.9999998803295952721994145-0.2886748978720895829872101 i$ & $1.2(-14)$ & $2.7(-7)$ & & $1.553078329(+1)$ & \\
\hline & & $1.0000000000004361507326680-0.2886751345951340174169557 i$ & $4.9(-26)$ & $5.4(-13)$ & & $1.555033068(+27)$ & \\
\hline & 3 & $1.0000000000000000000000000-0.2886751345948128822545744$ & $2.3(-98)$ & $3.7(-44)$ & 6.3558 & $1.469994999(+25)$ & \\
\hline \multirow{3}{*}{$O M 1$} & 1 & $1.0000277278805488765899181-0.288749982874054875236481 i$ & $1.1(-9)$ & $8.0(-5)$ & & $1.818924451(+6)$ & \multirow[t]{3}{*}{$6.861516768(+183)$} \\
\hline & 2 & $1.0000000000000000000000000-0.2886751345948128822545744 i$ & $2.7(-62)$ & $4.1(-31)$ & & $2.464971501(+2)$ & \\
\hline & 3 & $1.0000000000000000000000000-0.2886751345948128822545744 i$ & $4.3(-120)$ & $5.1(-60)$ & 1.0992 & $6.861516768(+183)$ & \\
\hline \multirow{4}{*}{$O M 2$} & 0 & $1.05-0.28 i$ & $4.2(-4)$ & $5.1(-2)$ & & & \multirow{4}{*}{$4.325592638(+138)$} \\
\hline & 1 & $1.0000937235784843654998272-0.2890812172236638332546179 i$ & $2.9(-8)$ & $4.2(-4)$ & & $9.507228477(+6)$ & \\
\hline & & $1.0000000000000000000000120-0.2886751345948128822545714 i$ & $2.5(-47)$ & $1.2(-23)$ & & $1.357758048(+4)$ & \\
\hline & 3 & $1.0000000000000000000000000-0.2886751345948128822545744 i$ & $9.2(-91)$ & $2.4(-45)$ & 1.1123 & $4.325592638(+138)$ & \\
\hline \multirow{2}{*}{$O M 3$} & 0 & $1.05-0.28 i$ & $4.2(-4)$ & $5.1(-2)$ & & & \multirow{2}{*}{$8.444513066(+136)$} \\
\hline & 3 & $1.0000000000000000000000000-0.2886751345948128822545744 i$ & $4.3(-90)$ & $5.1(-45)$ & 1.1229 & $8.444513066(+136)$ & \\
\hline
\end{tabular}

errors difference between two consecutive iterations but have also larger asymptotic error constant among other methods (OM2 and OM3) of same order which can be seen in Table 5. Such type of behavior can also seen in all the given tables except Table 2 wether you compare iterative methods of same order or different order of convergence.

\section{Conclusions}

In this manuscript we have designed the first, as far as we know, class of optimal eighth-order iterative methods for solving nonlinear equations with multiple roots, with known multiplicity $m$. The analysis of the local convergence has been made, proving the order eighth under standard assumptions regarding the nonlinear function whose zeros we are searching for. Some particular cases have been stated and their performance (compared with that of some known schemes) have been studied by using two different tools: the stability analysis provided by the dynamical planes associated to each numerical method applied on several real-life problems, whose behavior in the complex plane gives us information about the sensitivity of the methods to the initial guesses used; also, the classical numerical performance with high-precision calculation and the estimation of the residuals and order of convergence.

It has been proved that our proposed methods are efficient for determining multiple roots of nonlinear equations and are better with other well recognized efficient sixth-order iterative methods in all the considered problems analyzed. The proposed iterative methods not only have higher order of convergence, but also a stable behavior in the complex plane, with global convergence to the multiple root in many cases or wide areas of convergence in the rest of them. In addition, our methods have not only minimum residual error corresponding to considered test function $f$ but also have smaller error difference between two consecutive iterations. Moreover, minimum error between the consecutive iterations corresponding to the considered functions belongs to our proposed iterative methods. Hence, we confirm that our methods converge faster towards required zero of the corresponding function as compared to other existing methods, being in general, very stable.

Acknowledgments: The authors thank to the anonymous referees for their useful comments and suggestions to improve the final version of the manuscript.

\section{References}

1. Kung, H.T., Traub, J.F.: Optimal order of one-point and multipoint iteration. J. Assoc. Comput. Mach. 21 643-651 (1974)

2. Ostrowski, A. M.: Solution of equations and systems of equations. Academic Press, New York (1960)

3. Traub, J.F.: Iterative Methods for the solution of equations. Prentice-Hall, Englewood Cliffs (1964)

4. Petković, M.S., Neta, B. Petković, L.D., Džunić, J.: Multipoint methods for solving nonlinear equations. Academic Press (2013).

5. Behl, R., Cordero, A., Motsa, S.S., Torregrosa, J.R.: On developing fourth-order optimal families of methods for multiple roots and their dynamics. Appl. Math. Comput. 265(15) 520-532 (2015) 
6. Behl, R., Cordero, A., Motsa, S.S., Torregrosa, J.R., Kanwar, V.,: An optimal fourth-order family of methods for multiple roots and its dynamics, Numer. Algor. 71(4) 775-796 (2016)

7. Chicharro, F.I., Cordero, A., Torregrosa, J.R.: Drawing dynamical and parameters planes of iterative families and methods. The Scientific World Journal, 2013, Article ID 780153, 11 pages, (2013)

8. Li, S., Liao, X., Cheng, L.: A new fourth-order iterative method for finding multiple roots of nonlinear equations. Appl. Math. Comput. 215 1288-1292 (2009)

9. Li, S., Cheng, L., Neta, B.: Some fourth-order nonlinear solvers with closed formulae for multiple roots. Comput. Math. Appl. 59 126-135 (2010)

10. Neta, B., Chun, C., Scott, M.: On the development of iterative methods for multiple roots. Appl. Math. Comput. 224 358-361 (2013)

11. Sharma, J.R., Sharma, R.: Modified Jarratt method for computing multiple roots. Appl. Math. Comput. 217 878-881 (2010)

12. Zhou, X., Chen, X., Song, Y.: Constructing higher-order methods for obtaining the multiple roots of nonlinear equations. Comput. Appl. Math. 235 4199-4206 (2011)

13. Zhou, X., Chen, X., Song, Y.: Families of third and fourth order methods for multiple roots of nonlinear equations. Appl. Math. Comput. $2196030-6038$ (2013)

14. Neta, B.: Extension of Murakami's high-order non-linear solver to multiple roots. Int. J. Comput. Math. 87(5) 1023-1031 (2010)

15. Geum, Y.H., Kim, Y.I., Neta, B.: A class of two-point sixth-order multiple-zero finders of modified double-Newton type and their dynamics. Appl. Math. Comput. $270387-400(2015)$

16. Geum, Y.H., Kim, Y.I., Neta, B.: A sixth-order family of three-point modified Newton-like multiple-root finders and the dynamics behind their extraneous fixed points. Appl. Math. Comput. 283 120-140 (2016)

17. Artidiello, S., Cordero, A., Torregrosa, J.R., Vassileva, M.P.: Two weighted eight-order classes of iterative root-finding methods. Int. J. Comput. Math. 92(9) 1790-1805 (2015)

18. Shacham, M.: Numerical solution of constrained nonlinear algebraic equations. Int. J. Numer. Method Eng. 23 1455-1481 (1986)

19. Cordero, A., Torregrosa, J.R.: Variants of Newton's method using fifth-order quadrature formulas. Appl. Math. Comput. 190 (1) 686-698 (2007) 